\title{
Designing Modular Robotic Playware
}

\author{
Lund, Henrik Hautop; Marti, Patrizia
}

Published in:

18th IEEE International Symposium on Robot and Human Interactive Communication

Link to article, DOI:

10.1109/ROMAN.2009.5326286

Publication date:

2009

Document Version

Publisher's PDF, also known as Version of record

Link back to DTU Orbit

Citation (APA):

Lund, H. H., \& Marti, P. (2009). Designing Modular Robotic Playware. In 18th IEEE International Symposium on Robot and Human Interactive Communication: Ro-Man '09 (pp. 115-121). IEEE.

https://doi.org/10.1109/ROMAN.2009.5326286

\section{General rights}

Copyright and moral rights for the publications made accessible in the public portal are retained by the authors and/or other copyright owners and it is a condition of accessing publications that users recognise and abide by the legal requirements associated with these rights.

- Users may download and print one copy of any publication from the public portal for the purpose of private study or research.

- You may not further distribute the material or use it for any profit-making activity or commercial gain

- You may freely distribute the URL identifying the publication in the public portal

If you believe that this document breaches copyright please contact us providing details, and we will remove access to the work immediately and investigate your claim. 


\title{
Designing Modular Robotic Playware
}

\author{
Henrik Hautop Lund, Patrizia Marti
}

\begin{abstract}
In this paper, we explore the design of modular robotic objects that may enhance playful experiences. The approach builds upon the development of modular robotics to create a kind of playware, which is flexible in both set-up and activity building for the end-user to allow easy creation of games. Key features of this design approach are modularity, flexibility, and construction, immediate feedback to stimulate engagement, activity design by end-users, and creative exploration of play activities. These features permit the use of such modular playware by a vast array of users, including disabled children who often could be prevented from using and taking benefits from modern technologies. The objective is to get any children moving, exchanging, experimenting and having fun, regardless of their cognitive or physical ability levels. The paper describes two prototype systems developed as modular robotic tiles, and discusses the challenges and opportunities of this modular playware when used by children with different cognitive abilities.
\end{abstract}

\section{INTRODUCTION}

$\mathrm{I}$ this paper we describe a vision for the design of modular robotic technologies that combine constructive and sensorimotor play with creative and active participation of children regardless their specific abilities. Active games with physical involvement as well as building and creating outdoor fun and play in group or individually may all be supported by modular playware technologies that can not only help users reap the physical benefits of exercise, but also provides opportunities for them to learn, share, express feelings, set goals, and function independently. Playware is defined as intelligent hardware and software which aim at the creation of play and playful experiences.

The specific kind of modular robotic playware we present in this paper support play providing modular objects that are visible, manipulable, sharable and interactive and imply construction, active participation, creativity for assembling, mastery of the parts by the users who play with them. These technologies allow a range of play from simple exercise play up to construction play requiring sensory-motor skills as well as coordination/ manipulation of objects, acceptance of

Manuscript received March 15, 2009.

Henrik Hautop Lund is with Centre for Playware, Technical University of Denmark, Building 325, 2800 Kgs. Lyngby, Denmark (e-mail: hhl@playware.dtu.dk).

Patrizia Marti, is with the Communication Science Department, University of Siena, Siena 53100 Italy (phone: +39 0577234744; e-mail: marti@unisi.it). influences/inputs of others, turn taking, interdependency and collaboration. With such kind of play activities users can experience the pleasure of putting a "productive thought" into action, and can acquire and refine thinking and manipulation skills while using all their senses: the way toward complex forms of learning and social skill acquisition.

The concept that embraces this vision is today manifested in form of Tiles, modular units which can be assembled and are able to communicate with each other and to provide different kinds of feedback (light and sound). The different tile-components can support different play activities and can be set up by the user by physical (re)-programming or programming by examples.

\section{RELATED WORK ON INTERACTIVE TILES}

The concept of interactive tiles has been explored in different research projects and developed as commercial products along the last few years. Examples of interactive tiles are Sony DataTiles, tiles augmented by dynamic graphical information when they are placed on a sensorenhanced flat panel display [18]; Z-Tiles, a floor made from networked sensor tiles integrating pressure sensors connected to an embedded computer [19]; U-Texture, a selforganizable panel that can change its own behavior autonomously through recognition of its location, its inclination, and surrounding environment [15]; interactive MEDIATE wall, a floor surface developed for autistic children that reacts to movements generating feedback [17], and commercial products such as Twall from ExergameFitness, a touch surfaces for fitness, and LightSpace from LightSpace Corporation, a pressure sensitive surface able to detect location, movement and density of players to give a realistic gaming experience.

Most of these interactive surfaces, in particular walls and floors are all quite static set-ups that do not allow for the user to perform physical reconfigurations at run-time in an easy way and restrict the possible activities to pre-defined play on pre-defined surfaces.

\section{DESIGN FEATURES}

In response to the somewhat static nature of much related work, we will present a design approach that leads to flexible, interactive play tools for both sensorimotor and constructive play activities as designed by the end-users themselves. It is our belief that the flexibility obtained through distributed and modular playware holds many advantages for developing engaging and inclusive games and play. Therefore, the design approach outlines the principles 
that we suggest for the creation of flexible, modular play tools. The principles include the design of playware based upon modularity and flexibility, tangibility and immediate feedback to stimulate engagement, construction and physical movement, end-user activity design and inclusive games design.

\section{A. Playware}

The modular play tools that we develop are designed as playware. Playware is intelligent hardware and software which aim at the creation of play and playful experiences amongst users of all ages [9, 10]. Playware research and development seeks to understand play dynamics ${ }^{1}$ and implement them in play tools. Playware is of course not the only type of products which can create play, but we believe that digital technology contains new and expanded possibilities, e.g. when developed with modern artificial intelligence. Playware-tools are tools with a "behaviour" that initiates play force (e.g. a motion, in the case of sensorimotor play) via interaction. This is the basis for the play dynamic to emerge through which the users are brought into a state of playing.

The modern artificial intelligence is used to design behaviours of the play tools. The understanding of play dynamics can help guiding this design of behaviours to be used specifically to create playful and motivating tools for a variety of play interactions, well-knowing that there are both similarities and differences in the play dynamics of different users, environments and activities.

Indeed, the modern artificial intelligence technology supports the development of playware by providing means for creating adaptive play tools. We advocate here that for the sensorimotor and constructive play, especially modular robotics provides the possibility, through synthesis, to implement, test and understand play dynamics as modular robot technology gives way for the creation of physical interaction with (flexible) intelligent tools, which are able to react to the players' actions in a suitable manner.

\section{B. Modularity}

The modular robotic concept demands the availability of robotic modules with certain properties. Each robotic module needs to have a physical expression and should be able to process and communicate with its surrounding environment. The communication with the surrounding environment can be through communication to neighbouring robotic modules and/or through sensing or actuation. A modular robot is constructed from many robotic modules.

The modular robotics approach is to some extend inspired by the behaviour-based robotics approach [1], though the modular robotics approach builds on the belief that behaviour-based systems can include not only the coordination of primitive behaviours in terms of control units, but also include coordination of primitive behaviours in terms of physical control units. We can imagine a physical

\footnotetext{
${ }^{1}$ A play-dynamic is the dynamic effect of the play-force which affects the player by placing this person in a state of playing.
}

module being a primitive behaviour. Thereby, the physical organisation of primitive behaviours will (together with the interaction with the environment) decide the overall behaviour of the system. Hence, in a similar way to the control of robot behaviours by the coordination of primitive behaviours, we can imagine the overall behaviour of a robotic artefact to emerge from the coordination of a number of physical robotic modules that each represents a primitive behaviour.

The modular robotic concept is known mainly as selfreconfigurable robotic systems such as M-TRAN [13], ATRON [16], Superbot [20], etc., in which the modules can reassemble autonomously. In these self-reconfigurable modular robotics systems, the focus is on creating flexible and adaptive systems that respond to different environmental conditions and tasks by autonomously making run-time changes of the physical shape of the robotic system. On the other hand, we can view user-configurable modular robotic systems as modular robotic systems, where the user at runtime will make the physical rearrangement of the robotic modules. Some user-configurable modular robotic systems will allow the user to make the physical rearrangement of modules for setting up activities, while other userconfigurable modular robotic systems will allow the user to make the physical rearrangement as part of the activity (e.g. for play, learning, rehabilitation).

\section{Flexibility}

Both modalities of user-configurable modular robotic systems provide flexibility in the physical set-up and arrangement of the technological tool. It is the aim of such systems to allow for an easy way for end-users to make the physical and functional rearrangement. The design of such a flexible system demands a clear focus on how to create flexible and distributed technology.

Often, technological systems are created with a centralised, static set-up that does not allow for flexible rearrangement of the physical (and functional) arrangement of the systems. Even in the case of interactive tiles as presented in this paper, most other interactive surfaces are fairly static set-ups that most often only can be set-up in predefined physical configurations by a professional installation worker. So in such cases, play activities are most often limited to be performed on a pre-defined surface. There is little flexibility in terms of run-time development of the play activity based upon rearrangement of the interactive surface. The distributed system of tangible, attaching modules allow for a flexible use of the system by providing easy rearrangement (i.e. physical programming) possibilities.

In order to allow for flexible use of the different modular robotic devices, it becomes important to design and develop a distributed, generic, and versatile system (including communication protocol and framework) of the modular robotic devices, so that these modules can easily be added, removed, substituted, and rearranged. 


\section{Tangible interaction}

Tangible interaction refers to the technology enabled experience of physically manipulating objects [5], the material representations of information and the physical, conceptual and cultural constraints they embed. The mapping between the physical affordances of the objects with the digital components (different kinds of output and feedback) is a design and technological challenge. Indeed implementing the tangible interaction does not mean to simply augment physical objects with digital components. The physical properties of the objects serve as both representations and controls for their digital counterparts [6].

The concept of modular playware makes digital information directly manipulatable, perceptible and accessible through our senses by physically embodying it.

While playing with the modular playware, children can take advantage of the distinct perceptual qualities of e.g. the interactive tiles and this makes the interaction tangible, lightweight, natural and engaging. Interacting with tiles just means jumping over, pushing, assembling, touching physical objects and experiment a dialogue with them in a very direct and non-mediated way.

\section{E. Immediate Feedback}

In order to design the play experience in all its richness, feedback is a key features to guide children through the play activity. They expect to see the results of their actions immediately and if nothing happens after their input, they may give up and abandon the game.

Modular robotic playware creates rewarding games that can be easily understood and can promote fun. The design of such playware does not simply concentrate on the mechanics of the interface but also on immediate feedback that will keep children engaged.

User motivation and engagement are as important as task efficiency in modular robotic playware. Value is only attained if children spend time playing, enjoining and keeping their attention focused on the play activity.

In order to engage children in play, the feedback in modular robotic playware should be early and positive, immediate, explicit and understandable, continual and constructive as the child progressed through the activity.

\section{F. Construction and physical movement}

Constructive play combines sensorimotor practice with symbolic representation of ideas. Therefore it does not only stimulate the physical movement but also provide opportunities for make-believe and creative play activities. The modular robotic playware allows construction and deconstruction of assemblies to modify, enrich and adapt play activities to children with different abilities in different contexts.

Challenges in enabling constructive and creative play with modular robotic playware require that each part of the assembly is easy to understand at different levels: on the logical level (what can be done with the tiles, which tiles can go together and for what purpose of play), the functional level (how to use the tiles) and on the physical level (it must be possible to see what fits together, the system behaviour and what can be actually build or rebuild or modified).

\section{G. End-user activity design}

The importance of physical action as an active component of play activities and the direct physical interaction with the world are a key constituting factor not only of cognitive development during childhood but also of the design of play activities. In modular robotic playware, the play activity is designed by the end user through physical manipulation and shape configuration of modules.

This way of designing games by showing the modules the correct solution and by assembling them in different shapes has some similarities with programming by example [14] and physical programming [12]. The user can define specific patterns of modules and these modules initiate exploring their relative position. Changing the physical configuration of the modules results in changing the play activity.

Further, inspired by the body-brain discussion of modern artificial intelligence (e.g. [7]), it is possible to design systems that allow the user to modify the physical shape of the assembly of modules in order to change the playful game and interaction at run-time. The software game implementation may be fixed in the modules, and the change of the physical arrangement of the modules will enforce different kinds of physical interactions. It is the physical rearrangement of the modules that changes the game/interaction modalities (and not a change in SW).

\section{H. Inclusive games}

Modular robotic playware aims to get also disabled children moving, exchanging, experimenting and having fun, regardless of their cognitive or physical ability levels. Indeed our modular robotic tiles have been successfully used in spontaneous play activities involving disable children and their fully able brothers or friends.

Modularity, flexibility, immediate feedback and tangible activity design make such kind of playware adaptable to different physical and cognitive abilities. The adaptation can be performed at run time by the system itself or by the user as the activity progresses. For example tiles can be used in the swimming pool to support play in the water. Indeed water is an excellent medium to address the physical, cognitive, and psycho-social needs of children with a wide range of impairments. In the water they can not only increase their range of motion and coordination but also improve their independence, confidence and sensory processing. Moreover some quality of the feedback like pace and duration can be adapted to the child responsiveness and still the play activity maintains its characteristics to be demanding (requiring a complete commitment by the player), progressive (becoming gradually and increasingly complex) and fun.

\section{MODULAR ROBOTIC PLAYWARE: TWO PROTOTYPE SYSTEMS}

In what follows we describe two different implementations of the concept: the "Modular robotic tiles" modular tiles to support physical play on horizontal and 
vertical surfaces and the "Active surfaces", modular floating tiles to support construction play in the water.

\section{A. Modular robotic tiles}

We developed modular robotic tiles according to the key features of the design approach described above, in order to create a modular playware with the possibility for users to modify the physical shape and make easy setup for a variety of play activities. Further, we designed the modular system with the possibility of exclusion of external host computer, with self-contained energy source, with wireless communication (local and global), and with different games.
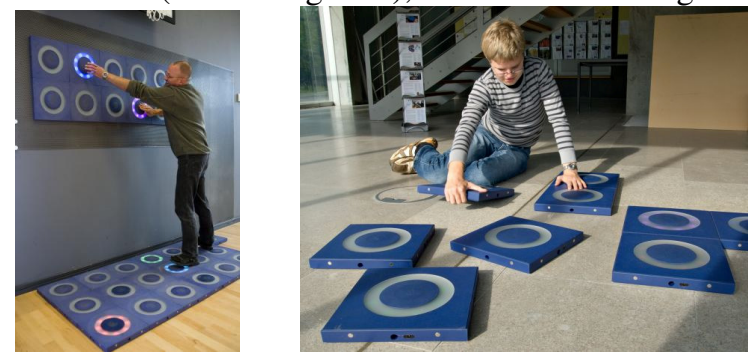

Fig. 1. The modular robotic tiles for sensorimotor play (left) and for construction play (right).

The system is composed of a number of modular robotic tiles which can attach to each other to form the overall system. Each tile has a quadratic shape (measuring $300 \mathrm{~mm} * 300 \mathrm{~mm} * 33 \mathrm{~mm}$ ), see Fig. 1. It is molded in polyurethane. In the center, there is a circular dent (diameter $200 \mathrm{~mm}$ ) which has a raised platform (diameter $63 \mathrm{~mm}$ ) in the centre. The dent can contain the circular printed circuit board with the electronic components. At the center of each of the four sides of the quadratic shape, there is a small tube (diameter $16 \mathrm{~mm}$ ) through which infra-red (IR) signals can be emitted and received (from neighboring tiles). Small magnets are placed on each side of the tiles. The magnets on the back provide opportunity for a tile to be mounted on a magnetic surface (e.g. wall), and the magnets on the sides provide opportunities for the tiles to attach to each other. The magnets ensure that when two tiles are put together they will become aligned by the magnetic forces, which is important for ensuring that the tubes on the two tiles for IR communication are aligned and it helps the end-user in making the correct assembly in a simple, easy and understandable manner. On one side of the tile, there is also a small hole for a battery charging plug.

There is a small groove on the top of the wall of the circular dent, so a circular cover (diameter $210 \mathrm{~mm}$ ) can be mounted on top of the dent. The cover is made from a circular transparent satinice plate and a polyurethane circle in the centre.

A force sensitive resistor (FSR) is mounted as a sensor on the center of the raised platform underneath the circular cover. This allows analogue measurement of the force exerted on the top of the cover. There are three NIMH AA rechargeable batteries on top of the PCB. A 2 axis accelerometer is mounted, e.g. to detect horizontal or vertical placement of the tile. Eight RGB light emitting diodes are mounted with equal spacing in between each other on a circle on the PCB, so they can light up underneath the transparent satinice circle. There is a radio communication add-on PCB with a XBee radio communication chip.

The modular robotic tiles can easily be set up on the floor or wall within one minute. The tiles can simply attach to each other with the magnets, and there are no wires. With the accelerometer, the tiles can register whether they are placed horizontally or vertically, and by themselves make the software games behave accordingly.

Also, the tiles can be put together in groups, and the groups of tiles may communicate with each other wireless (radio). For instance, a game may be running distributed on a group of tiles on the floor and a group of tiles on the wall, demanding the user to interact physically with both the floor and the wall (see Fig. 1, left).

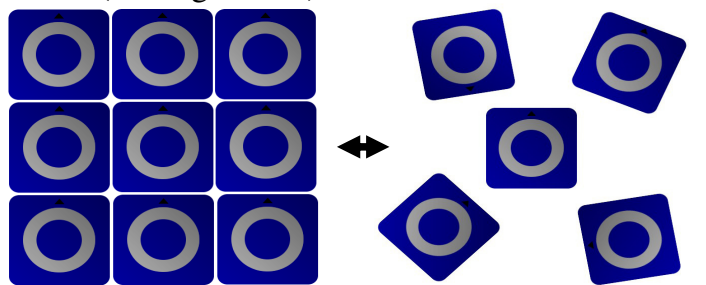

Fig. 2. The modular robotic tiles can be physically reconfigured by any user at run-time, e.g. as one cluster (left) or more clusters of size down to one tile for each cluster (right).

The tiles are developed according to the design approach to provide a flexible possibility to create a variety of playful applications for a variety of end-users. For instance, we designed applications for playful physical activities, which were installed in 6 kindergartens, schools and youth clubs in Odense, Denmark, and we made interactive soccer games with the tiles for children's entertainment at stadium during Danish national football league matches.

Among many different games, we implemented the colour race game, where the child has to chase a given colour, which jumps around on the surface of tiles. The modularity of the tiles provides flexibility for creating tangible interaction. If the child (or teacher) wants playful interaction with hands, she places the tiles on a wall so that the child needs to play with the hands, or if she wants interaction with the feet, she may place the tiles on the floor. Or she can choose a combination of some tiles on the ground and some tiles on the wall for playful interaction with both hands and feet. For the soccer game, a light is travelling around on the tiles, and the child has to hit the travelling light with the ball, which will light up all the tiles to indicate that a goal was scored - the game becomes an interactive version of playing soccer up against a wall (Fig.3, right).
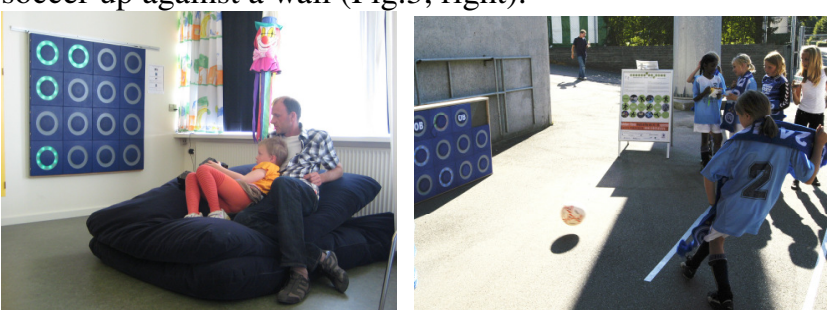

Fig. 3. A child and her father in the multi-sensory room (left), and children playing soccer with the modular robotic tiles (right). 
As a result of the design, other motivating play activities may easily be set up for children (and adult) e.g. for sensorimotor play such as stepper games, reaching games, dancing games, and more cognitive sensorimotor games such as Memory and Simon says.

Therefore, the design approach also allowed us to quickly reconfigure the modular robotic tiles to be used for playful cognitive construction games (see fig. 1, right). We used a set of 15 tiles and the construction game called colour-mix. The basic idea is to mix colours in different ways, dependent on how the tiles are assembled. 3 tiles are predefined as source tiles respectively with the colours red, green and blue. The other 12 tiles are normal tiles, with the property that they can change their colours accordingly to their local neighbourhood. If a normal tile is connected to a red source tile, the normal tile will become red just as its neighbour but with a lower intensity. The source tiles never change their colour. If a blue source tile also is connected to the normal tile at the same time as a red source tile, the normal tile will blend the two colours to become a purple tile. A normal tile should always light up with a lower intensity than its neighbours colour intensity, which makes the colour spreading from a source tile decrease when the distance to a source tile increases.

For the colour-mix construction game, we used a distributed control approach, which is fairly straight-forward since every modular robotic tile is equipped with both communication and computation capabilities. The tiles can be moved around and connected to each other in any configuration. In this distributed environment it is very easy to make local changes based on the local environment. A tile can easily read neighbouring tiles' states, and thereby change its own state accordingly to some local rules. By not having a central server to administer the data flow between tiles, the stability of the application will not depend on the reachability of e.g. a master-tile or a host computer. Simple rules based on the local environment are easily implemented and the software on the individual tile can be kept simple. Also, the distributed control facilitates the emergence of new behaviours, when different rules are influencing each other. It is not always possible to predict what can emerge, and this is in the hands of the end-users construction.

Also, we designed the tiles to be used as part of a multisensory room at the HCA children's hospital in Denmark (see Fig. 3 left). In a similar manner to the applications described above, the multi-sensory room composed of the modular robotic tiles and other modular devices (e.g. I-BLOCK cubes, and sound system) engage the hospitalized children in physical activities, and should motivate to perform physical activities by providing immediate feedback (coloured light patterns, sound, vibration) based upon playful, physical interaction with the system. The children hospital pedagogues allow the children and possibly their parents into the multi-sensory room to interact with the playful robotic tiles to provide a calm, relaxing and joyful environment. A main finding of the tests conducted at a children's hospital, was that it was found to be very important to create feedback that was easily recognised by the users [8]. Indeed, the tests showed it to be crucial to create feedback that was easily and immediately recognised by the users, and it was found that the interaction was boring if the feedback was too implicit (subtle) and not well understood by the user [8]. The users appreciated an explicit immediate feedback (e.g. a clear and immediately recognisable sound or light pattern from touching a tile) because it was obvious and understandable, and did not require any a priori knowledge of the dynamics of the games.

The modularity, ease of use and the functionality of the modular playware devices suits well into scenarios such as playful multisensory rooms, because they provide coloured light, sound and possibly other kinds of feedback (e.g. vibration). The tiles are designed generic, which means that they can be augmented with other sensors or actuators. The physical form and the weight of the tiles is important to allow for easy rearrangement of the tiles for any child.

Our design guidelines lead us to construction of tiles that lend themselves as inclusive games technology in that the user groups for the applications may span an array of abilities. Indeed, the colour mix application was actually developed and performed with therapists and 7 autistic children in the autism home Bihuset in Denmark. We will explore and exemplify the design approach for inclusive games technology in further details with the design of active surface tiles below.

\section{B. Active surfaces}

Physical and cognitive rehabilitation of children with special needs poses important technological challenges and design opportunities. For example, therapists need to be able to react flexibly and fast to children's different abilities, and therapists and children need multiple modes of interaction, including physical ones. These demands play out most interestingly in aquatherapy, where we must also add communication and resource challenges.

Aquatherapy is a crucial part of cognitive and physical therapy. Children usually enjoy swimming, and being in the water decreases the effects of gravity, making activities involving balance and coordination more attainable for the child. In the water children with special needs can not only increase their range of motion and coordination but also improve their independence, confidence and sensory processing. The water is an excellent medium to address the physical, cognitive, and psycho-social needs of children with a wide range of impairments, including, for example, cerebral palsy, Down's syndrome, autism, neurological disorders, perceptual difficulties.

Aquatherapy is mainly achieved through games designed to improve motion and cognitive skills. For the therapists this is a highly demanding task. Games must be invented or modified on the fly to take advantage of the children's widely varying strengths. Moreover, whereas a child with cognitive difficulties might respond to verbal encouragement to put objects in an ascending order (e.g. of size), children with communication difficulties respond better to visual cues and specific tangible rewards than to verbal directions, creating a need for flexible multi-media stimulation. 
Currently the therapists' efforts are poorly supported, and there is a clear need to develop tools that support creative and situation sensitive therapy by being easily reconfigurable during the activity and adaptable to evolving situations, and by supporting a range of multi-media feedback.

1) The system

The Active Surfaces is a modular system consisting of floating tiles measuring $30 * 30 * 5 \mathrm{~cm}$ (Fig. 4). Children have to assemble the floating tiles into meaningful configurations defined by the therapist. Each tile is a resource constrained embedded system that communicates with the others over the six sides using only a low bandwidth short-range infrared link. Tiles can only communicate if they are close to each other. The output available to users is a set of light emitting diodes which provide feedback during the task and reward at the task completion.

The tiles support multiple games by having a simple physical appearance and multi-purpose programmable hardware. Magnets are placed on the four sides of each tile to make the tiles "snap" together when they are in close vicinity. A replaceable plastic cover is on the top of the tile also held in place by magnets. The image on the cover depends on the game. Inside each tile an embedded system uses infrared light to communicate with and detect the presence of other tiles. Two tiles can only communicate if they are close to each other. The main computational unit is the UNC20 module, which is an ARM7-based embedded system running uClinux at $55 \mathrm{MHz}$ with approximately $8 \mathrm{MB}$ ram. The UNC20 module communicates with a sideboard using a serial connection. The sideboard is responsible for controlling the infrared communication and the LEDs. The bandwidth of the infrared communication is approximately 600 bits per second [2].

The Active Surfaces prototypes support construction activities on different levels: i) on the logical level the therapist can define what the rules of the game are and what the purpose is; ii) on the functional level users can mark out the relations and the sequences and; iii) on the physical level users can define which patterns and connections can take place, to reach the goal of the game [3].

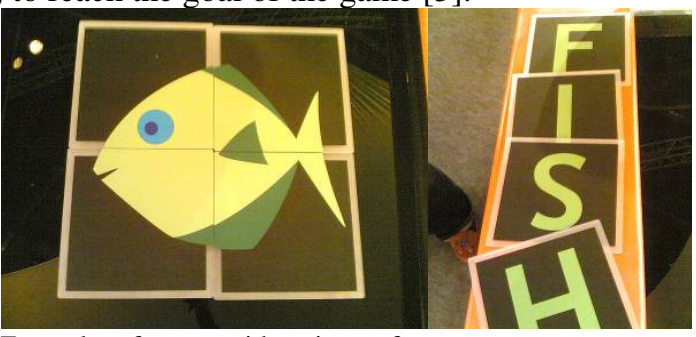

Fig. 4. Examples of games with active surfaces

Games with different logics have been created to support aquatherapy on a resource-constrained device like the tile [4]. For example, children with perceptual problems can be stimulated using the catch game. In this game a set of tiles are placed in the swimming pool and another tile is given to the child. When the game is started the child has to get her tile close to a glowing tile, "catching the light" within a certain timeframe. If she succeeds, another random tile will light up and she tries to catch that one. When she eventually fails to catch the light in time her tile will blink how many lights she caught. Other games like the scrabble game, where the child has to form words out of letters can be used for children with language problems, or sequence games like putting in a sequence tiles with images of increasing size, or matching colours, can be used for children with Down's Syndrome (Fig 4).

Active Surfaces have been experimented in different Italian institutions: the 'Le Scotte' Hospital in Siena, the Disabled Children Parents' Association in Siena and the D. Chiossone rehabilitation centre in Genova (Fig. 5).

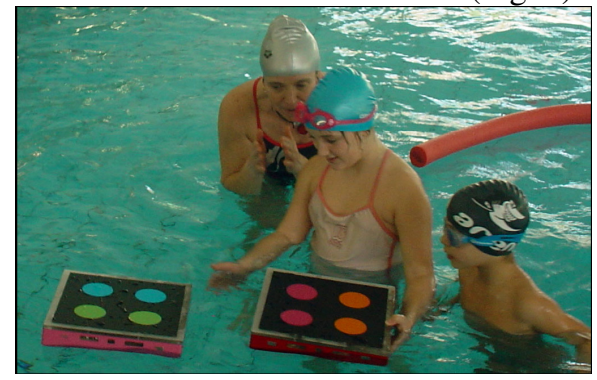

Fig. 5. Trials with active surfaces

In some therapeutic sessions, the system has been successfully used in spontaneous activities involving disable children and their fully able brothers or friends. In other cases children with mild cognitive delay and physical impairments were treated for attention and object manipulation tasks or the creation of logical sequences in the domino game. In all sessions, the children enjoyed the play activity. They tried out different construction games but also explored unusual combinations of the tiles like piling them one on top of the other. A remarkable result of the experimentation is observation of the emergence of the exploration of body schemes, like the trunk-shoulders coordination during the rotations, the left-right movements, the control of spatial relations among the floating objects (inside/outside, front/rear, top/bottom). The acquisition of the body schemes and the spatial relations among objects is a slow conquest for children not attributable to the simple mental access to these concepts. For children with multiple disabilities the acquisition of spatial concepts is enabled by the psycho-motoric activity and the experience of their body in the world. Active surfaces just stimulate such kind of exploration of our own body and its spatial relations with other objects. A second remarkable result of the trials is that the rehabilitation consisting of repetitive exercises is often considered by the children boring, gruesome and painful and for this reason children are reluctant or just refuse to practice the exercises.

With active surfaces disabled children accepted the physical competition of the construction games and collaborated with each other to their successful accomplishment. Also the therapists interviewed after the therapeutic sessions considered active surfaces to be an excellent substitute for traditional therapy because, though it requires body movements just like traditional therapy, it 
involves so much of distraction and fun that children ignore the discomfort [11]

\section{DISCUSSION AND CONCLUSIONS}

The two different kinds of tiles incorporate and exemplify the key features of the design approach. The modularity is obtained by the development of fully distributed and selfcontained modular robotic tiles, which allow the user to make both physical and functional construction. Thereby, the tiles can be used in a flexible manner by allowing the enduser to make changes in an easy and very fast manner, so that it is the end-user who can design the activity. The design focus on tangible interaction led to solutions such as utilising the material properties to facilitate understanding of behavioural characteristics and manipulable possibilities (as an example, magnetic forces of tiles "invisibly" helped the end-users assembling (aligning) the tiles correctly). By sensing the exerted force and providing coloured light (and sound) stimuli, the tiles can provide an immediate feedback. If this immediate feedback is explicit and provided as part of a playful interaction game (for both sensorimotor and construction play), we find that it motivates and stimulates engagement, as exemplified with the variety of applications presented above.

These key features further make it possible to create inclusive games which may be adapted to users with different physical and cognitive abilities. Indeed, the tiles were easily adopted by children with different cognitive abilities as exemplified above, but also by adults for physiotherapy and for elderly cardiac and stroke patients' physical rehabilitation (at the hospital Sygehus Fyn Svendborg, 2006-09). The design of modular playware allows this adaptation to happen automatic by software adaptation or by the users' easy physical construction with the tiles. Further, in these cases, the design focus on playware results in playful applications that motivate to perform rehabilitation activities.

In the future, we will explore the design approach further, for instance by utilising it to create playful, interactive and flexible modular playware for rehabilitation of handicapped children at Neema Craft in Iringa, Tanzania. Here, it is our belief that the design approach comes to its full value when allowing for easy and flexible adaptation to the local, contextual needs.

\section{ACKNOWLEDGMENTS}

The authors would like to thank colleagues in their respective research groups, Center for Playware at Technical University of Denmark and Communication Science Department at University of Siena. Their contributions were essential for allowing the authors to form the ideas and principles presented here.

\section{REFERENCES}

[1] Brooks, R.A. A robust layered control system for a mobile robot. IEEE Journal of Robotics and Automation, 2(1):14--23, 1986.
[2] Brønsted J., Grönvall E., Svensson Fors D., Palpability support demonstrated, The 2007 IFIP International Conference on Embedded and Ubiquitous Computing, Taipei, Taiwan, December 17 - 20, 2007.

[3] Grönvall, E., Marti, P., Pollini, A., Rullo, A., Active Surfaces: a novel concept for end-user composition, in G. Ghosh, D. Svanæs, "Changing Roles". Proceedings of NorCHI 2006, the Fourth Nordic Conference on Human Computer Interaction. October 16-18, 2006, Oslo.

[4] Grönvall E., Pollini A., Rullo A., Svensson D., Designing game logics for dynamic Active Surfaces, MUIA06, Espoo, Finland, 12 September 2006.

[5] Hornecker E., Buur J. Getting a grip on tangible interaction: a framework on physical space and social interaction. Proceedings of CHI 2006 (C) ACM, April 22-28, 2006, Montréal, Québec, Canada, 2006.

[6] Ishii, H. Ullmer, B. Tangible Bits: Towards Seamless Interfaces between People, Bits and Atoms. In Proceedings of CHI: Human Factors in Computing Systems. pp. 234-41, 1997.

[7] Lund, H. H., Hallam, J., and Lee, W.-P. Evolving Robot Morphology. In Proceedings of IEEE 4th International Conference on Evolutionary Computation. IEEE Press, NJ, 1997.

[8] Lund, H. H, Henningsen, A., and Nielsen, R. Modular Robotic System as Multisensory Room in Children's Hospital. In Sugisaka (Ed.) Proceedings of 14th International Symposium on Artificial Life and Robotics, Oita, ISAROB, 2009.

[9] Lund, H. H., and Jessen, C. Playware - Intelligent technology for children's play, Technical Report TR-2005-1, June, Maersk Institute, University of Southern Denmark, 2005.

[10] Lund, H. H., Klitbo, T., and Jessen, C. Playware Technology for Physically Activating Play, Artificial Life and Robotics Journal, 9:4, 165-174, 2005.

[11] Marti, P. Pollini, A. "Spazi di Gioco e Spazi di Cura: il Ruolo delle Tecnologie Interattive nel Trattamento delle Disabilità Motorie e Cognitive". In S. Besio (a cura di) Gioco e giocattoli per la disabilità motoria. Unicopli, Milano, In press.

[12] Montemayor, J., Druin, A., Farber, A., Simms, S., Churaman, W., D'Amour, A.: Physical programming: designing tools for children to create physical interactive environments. In: CHI '02: Proceedings of the SIGCHI conference on Human factors in computing systems, New York, NY, USA, ACM Press (2002) 299-306.

[13] Murata, S., E. Yoshida, A. Kamimura, H. Kurokawa, K. Tomita, and S. Kokaji (2002) 'M-TRAN: Self-Reconfigurable Modular Robotic System'. IEEE/ASME Transactions on Mechatronics 7(4), 431-441.

[14] Myers, B.A. Visual programming, programming by example, and program visualization: a taxonomy. SIGCHI Bull. 17(4) (1986) 59-66

[15] Ohsawa, R. Kohtake, N. Takashio, K. Tokuda, H. Software architecture for self-organizable universal boards. In Software Architectures for Self-Organization: Beyond Ad-Hoc Networking Workshop at The 3rd International Conference on Pervasive Computing (PERVASIVE 2005), May 2005.

[16] Ostergaard, E. H., Kassow, K., Beck, R. and Lund, H. H. Design of the ATRON lattice-based self-reconfigurable robot. Autonomous Robots 21(2), 165-183, 2006.

[17] Parés, N. Carreras, A. Soler, M. Non-invasive attitude detection for full-body interaction in MEDIATE, a multisensory interactive environment for children with autism. Proceedings of Vision, Modeling, and Visualization 2004 (VMV'04). Stanford, USA, 2004, $37-45$

[18] Rekimoto, J., Ullmer, B., Oba, H. DataTiles: A Modular Platform for Mixed Physical and Graphical Interactions, CHI2001, 2001.

[19] Richardson, B. Fernström, M. Network and Control Protocol for a Self-Organising Sensor Network. Proc. CCCT, 2003.

[20] Salemi, B., Moll, M. and Shen, W.-M.. SUPERBOT: A Deployable, Multi-Functional, and Modular Self-Reconfigurable Robotic System. In Proc. 2006 IEEE/RSJ Intl. Conf. on Intelligent Robots and Systems, Beijing, China, October 2006 